\title{
Association between infectious burden, socioeconomic status, and ischemic stroke
}

\section{Palm, Frederick}

2016-11

Palm , F , Pussinen , P J , Aigner , A , Becher , H, Buggle , F, Bauer , M F , Grond-Ginsbach , C , Safer , A , Urbanek , C \& Grau , A J 2016 , ' Association between infectious burden, socioeconomic status, and ischemic stroke ' , Atherosclerosis , vol. 254 , pp. 117-123 . https://doi.org/10.1016/j.atherosclerosis.2016.10.008

http://hdl.handle.net/10138/230922

https://doi.org/10.1016/j.atherosclerosis.2016.10.008

publishedVersion

Downloaded from Helda, University of Helsinki institutional repository.

This is an electronic reprint of the original article.

This reprint may differ from the original in pagination and typographic detail.

Please cite the original version. 


\title{
Association between infectious burden, socioeconomic status, and ischemic stroke
}

\author{
Frederick Palm ${ }^{\mathrm{a},}$, , Pirkko J. Pussinen ${ }^{\mathrm{b}}$, Annette Aigner ${ }^{\mathrm{c}}$, Heiko Becher ${ }^{\mathrm{c}, \mathrm{d}}$, \\ Florian Buggle ${ }^{a}$, Matthias F. Bauer ${ }^{\mathrm{e}}$, Caspar Grond-Ginsbach ${ }^{\mathrm{f}}$, Anton Safer ${ }^{\mathrm{d}}$, \\ Christian Urbanek ${ }^{\mathrm{a}}$, Armin J. Grau ${ }^{\mathrm{a}}$ \\ a Department of Neurology, Klinikum Ludwigshafen, Germany \\ ${ }^{\mathrm{b}}$ Oral and Maxillofacial Diseases, University of Helsinki, Finland \\ c Institute of Medical Biometry and Epidemiology, University Medical Center Hamburg-Eppendorf, Germany \\ ${ }^{\mathrm{d}}$ Institute of Public Health, University of Heidelberg, Germany \\ e Clinical Chemistry, Klinikum Ludwigshafen, Germany \\ ${ }^{\mathrm{f}}$ Department of Neurology, University of Heidelberg, Germany
}

\section{A R T I C L E I N F O}

\section{Article history:}

Received 5 August 2016

Received in revised form

4 October 2016

Accepted 4 October 2016

Available online 5 October 2016

\section{Keywords:}

Ischemic stroke

Infection

Periodontitis

Large vessel disease

Socioeconomic status

\begin{abstract}
A B S T R A C T
Background and aims: Infectious diseases contribute to stroke risk, and are associated with socioeconomic status (SES). We tested the hypotheses that the aggregate burden of infections increases the risk of ischemic stroke (IS) and partly explains the association between low SES and ischemic stroke.

Methods: In a case-control study with 470 ischemic stroke patients and 809 age- and sex-matched controls, randomly selected from the population, antibodies against the periodontal microbial agents Aggregatibacter actinomycetemcomitans and Porphyromonas gingivalis, against Chlamydia pneumonia, Mycoplasma pneumoniae (IgA and IgG), and CagA-positive Helicobacter pylori (IgG) were assessed.

Results: IgA seropositivity to two microbial agents was significantly associated with IS after adjustment for SES (OR 1.45 95\% CI 1.01-2.08), but not in the fully adjusted model (OR 1.32 95\% CI 0.86-2.02). By trend, cumulative IgA seropositivity was associated with stroke due to large vessel disease (LVD) after full adjustment (OR 1.88, 95\% CI 0.96-3.69). Disadvantageous childhood SES was associated with higher cumulative seropositivity in univariable analyses, however, its strong impact on stroke risk was not influenced by seroepidemiological data in the multivariable model. The strong association between adulthood SES and stroke was rendered nonsignificant when factors of dental care were adjusted for. Conclusions: Infectious burden assessed with five microbial agents did not independently contribute to ischemic stroke consistently, but may contribute to stroke due to LVD. High infectious burden may not explain the association between childhood SES and stroke risk. Lifestyle factors that include dental negligence may contribute to the association between disadvantageous adulthood SES and stroke.
\end{abstract}

(c) 2016 Elsevier Ireland Ltd. All rights reserved.

\section{Introduction}

Stroke, especially ischemic stroke (IS), is a multifactorial disease with numerous contributing risk factors. These risk factors are target of preventive strategies attempting to mitigate the future burden of stroke. Current risk factor models may explain about $90 \%$ of ischemic stroke occurrence [1]. However, some epidemiological

\footnotetext{
* Corresponding author. Department of Neurology, Städtisches Klinikum Ludwigshafen, Bremserstr. 79, 67063 Ludwigshafen a. Rh., Germany.

E-mail address: palmf@klilu.de (F. Palm).
}

aspects of stroke may not be sufficiently explained yet [2-4].

Infectious and inflammatory diseases, as well as unfavorable socioeconomic conditions have been described as contributors to the risk of stroke [4-7]. Different chronic infections have been found to be associated with stroke, including clinical periodontitis, infections with the periodontal pathogens Porphyromonas gingivalis (Pg) and Aggregatibacter actinomycetemcomitans (Aa), infections with Helicobacter pylori (Hp), and particularly with $\mathrm{Hp}$ strains carrying the cytotoxin-associated gene A (CagA) [8-11]. Chlamydia pneumonia (Cp), Mycoplasma pneumonia (Mp), and Legionella pneumophila (Lp) are as well among those microbial agents whose link with stroke has been discussed [12]. However, the results of 
studies investigating single agents are ambiguous [13]. A current hypothesis suggests that the aggregate burden of microbial agents to which an individual has been exposed to during his or her whole lifetime, rather than single pathogens, increases the risk of stroke ("infectious burden concept") [5]. In a recent analysis of the Northern Manhattan Study, no association was found between the risk of stroke and any of the five serologies to common infections (Cp, Hp, Cytomegalovirus, Herpes Simplex Virus 1 and 2). However, a derived infectious burden index showed an association with stroke [14].

Socioeconomic status (SES) is negatively associated with the risk of stroke [15]. In a case-control study, we recently showed that SES during childhood and adulthood are each independently linked to stroke risk [16]. Several chronic infectious diseases such as periodontitis and infection with $\mathrm{Hp}$ are more common in people with disadvantageous social conditions [17]. Thus, it seems likely that chronic infections contribute to the association between socioeconomic conditions and stroke, together with other factors such as a health neglecting lifestyle.

Based on the data from the same case-control study, we tested the hypotheses that the infectious burden (=aggregate number of seropositivities to selected infectious agents) is associated with the risk of IS and that chronic infections and elements of a health neglecting lifestyle contribute to the association between SES during childhood, adolescence, and adulthood and the risk of IS.

\section{Patients and methods}

The "GENESIS" study is a case-control study with 470 first-ever ischemic stroke (FEIS) cases ( $40 \%$ women, mean age $66.5 \pm 10.8$ years; $60 \%$ men, $65.5 \pm 10.7)$ and 809 age- and sex-matched controls (41.8\% women, $66.4 \pm 11.1 ; 58.2 \%$ men, $67.9 \pm 9.5)$, randomly selected from the general population. The target population consists of individuals aged $18-80$ years, living in the city of Ludwigshafen am Rhein in South-West Germany. „GENESIS“ was established within the framework of the Ludwigshafen Stroke Study (LuSST), a population-based stroke registry that started on January 1st, 2006, using standard definitions and multiple overlapping methods of case-ascertainment in order to identify all cases with incident stroke or transient ischemic attack among the population of Ludwigshafen (163.340 inhabitants on December 31, 2009). A detailed description of LuSSt and the „GENESIS“ study have been published recently $[16,18]$. The study was approved by the ethics committee of the Landesärztekammer Rheinland-Pfalz (837.333.05(4991)).

\subsection{Inclusion and exclusion criteria}

Inclusion criteria besides age and permanent residency in the study area were Caucasian ethnicity and written informed consent to study participation. Inclusion criterion for cases was the diagnosis of a FEIS based on an acute neurological deficit lasting $>24 \mathrm{~h}$ with no other reason than cerebral ischemia. All cases received a cerebral CT or MRI. Exclusion criteria for both cases and controls included any previous stroke, myocardial infarction within 90 days, dementia, severe aphasia, insufficient understanding of the German language or any other relevant communication barrier and severe disability that precludes interview participation.

\subsection{Recruitment}

For recruitment of controls, a random sample of Ludwigshafen residents was drawn from the population registry including name, age, sex and address. Subsamples were consecutively taken to match the age and sex distribution of cases. Those selected received invitation letters with detailed information on the study and request for their participation. The participation rate for controls was $46.6 \%$. The cases included incident stroke cases from the LuSSt registry. According to the study protocol only in-patients at the Klinikum Ludwigshafen were asked for participation in "GENESIS", since only few patients in the area did not attend this hospital. This group represents about $89 \%$ of all cases in LuSSt. The participation rate for cases was $73.7 \%$.

\subsection{Data collection and laboratory tests}

Cases and controls were interviewed by trained interviewers using a standardized questionnaire. We collected data on age, sex, anthropometric measures, previous diseases, and previous visits to a dentist as markers of health behavior, number of teeth, smoking, alcohol intake, physical activity, dietary patterns, medication and social history. In both, cases and controls, blood pressure was measured after 5 min of resting, a 12-lead electrocardiogram and a Duplex-sonography of brain supplying arteries were performed. Venous blood samples were taken in cases and controls.

Serum was separated by low-speed centrifugation and stored at $-70{ }^{\circ} \mathrm{C}$ until analysis. Serum levels of IgA and IgG antibodies against $A a$ and $P g$ were determined by multi-serotype-ELISA as previously described [19]. Serum levels of IgG antibodies against $H p$ and of IgG and IgA antibodies against $C p$ and $M p$ were assessed by using commercial enzyme immunoassays (Enzygnost ${ }^{\circledR}$ and Novagnost $^{\circledR}$ Siemens Healthcare) at the Institute of Clinical Chemistry, Klinikum Ludwigshafen.

\subsection{Definition of variables}

Cardiovascular risk factors were defined according to current national and international guidelines and have been described in detail [20]. A detailed presentation of the SES measures has been published recently [16]. Etiological subtypes of IS were ascertained using modified TOAST criteria (Trial of ORG 10172 in Acute Stroke Treatment) as previously described [20]. The category "large vessel disease" (LVD) included "probable atherothrombotic stroke" (AT) and stroke due to "large artery atherosclerosis" (LAA). In 159 cases, etiology of IS was classified as LVD (19.7\% of all cases).

\subsection{Statistical analysis}

We report absolute and relative frequencies of risk factors, SES and seropositivities to infectious agents by case-control status. Infectious burden was calculated as cumulative IgA-positivity, cumulative IgG-positivity, and aggregated IgG/IgA-positivity. Odds ratio (OR) estimates and 95\% confidence intervals (CI) are presented based on conditional logistic regression models, conditioned on age (2-year-age groups) and sex, and adjusted for risk factors, SES, and the respective other infection indicators. Associations between the number of seropositivities to infectious agents and SES in childhood, adolescence, and adulthood were investigated by the Jonckheere-Terpstra trend test. To control for a potential bias owing to a lower response of controls from more disadvantaged social background, we performed a sensitivity analysis, comparing school education data in our control group with those of the general population received from the municipal statistical office. Although individuals with low school education were moderately underrepresented in our control group, results regarding socioeconomic factors as stroke risk factor were altered only to a minor degree by adjustment for a differential response rate in controls [16]. For all analyses, the statistical software $\mathrm{R}$ was used, conditional logistic regression estimates were obtained through the $\mathrm{R}$ package survival, the Jonckheere-Terpstra trend test 
from the R package clinfun [21-23].

\section{Results}

Distribution of traditional risk factors, lifestyle risk factors, socioeconomic risk score and dental risk factors in cases and controls is shown as a supplementary table as previously published [16]. FEIS cases showed higher rates of seropositivity regarding IgA antibodies against $A a$ and IgG antibodies against CagA-positive strains of $\mathrm{Hp}$ but lower rate of seropositivity to IgG antibodies against $\mathrm{Mp}$ in analyses adjusted for age and sex only. Cumulative IgAseropositivity against 2 bacterial strains was associated with higher stroke risk, but cumulative IgG or combined IgA/IgG seropositivity was not (Table 1 ).

Adjustment for SES in childhood, adolescence and adulthood and further adjustment for cardiovascular risk factors and dental parameters had mostly small effects on the risk estimates of the immunological variables (Fig. 1A). The association between $\mathrm{Hp}$ and IS was weakened and rendered nonsignificant by adjustment for SES. IgA seropositivity to two microbial agents was significantly associated with IS after adjustment for SES (OR $1.4595 \% \mathrm{CI}$ 1.01-2.08). Fully adjustment rendered effect of IgA-seropositivity to two microbial agents on stroke risk nonsignificant (OR 1.32 95\% CI 0.86-2.02).

Distribution of risk factors and data on SES was reported previously [16]. Data on dental health showed that patients suffered more often from advanced tooth loss (8-14 teeth remaining) and used dental health care service less than controls (Table 2).

When analyzing cases with LVD separately, IgA-seropositivity for $\mathrm{Aa}$ (OR 1.68; 95\% CI 1.15-2.46) and Cp (OR 1.89; 95\% CI 1.14-3.13), cumulative IgA-seropositivity for 2 (OR 2.14; 95\% CI

\section{Table 1}

Distribution, odds ratios and 95\%-confidence intervals of serological testing and aggregate number of seropositivities to infectious agents for all ischemic stroke cases.

\begin{tabular}{|c|c|c|c|c|c|c|}
\hline & Controls & $\%$ & Cases & $\%$ & Crude $\mathrm{OR}^{\mathrm{a}}$ & $95 \% \mathrm{CI}$ \\
\hline \multicolumn{7}{|c|}{ Mycoplasma pneumonia } \\
\hline $\operatorname{Ig} A$ & 24 & 2.98 & 15 & 3.22 & 1.04 & $0.53-2.06$ \\
\hline $\operatorname{IgG}$ & 445 & 55.28 & 232 & 49.79 & 0.74 & $0.59-0.95$ \\
\hline \multicolumn{7}{|c|}{ Porphyromonas gingivalis } \\
\hline $\operatorname{Ig} A$ & 434 & 53.91 & 253 & 54.88 & 1.06 & $0.83-1.35$ \\
\hline IgG & 594 & 73.79 & 344 & 74.62 & 1.08 & $0.81-1.42$ \\
\hline \multicolumn{7}{|c|}{ A. actinomycemtemcomitans } \\
\hline $\operatorname{Ig} A$ & 220 & 27.33 & 146 & 31.67 & 1.33 & $1.02-1.73$ \\
\hline $\operatorname{IgG}$ & 244 & 30.31 & 118 & 25.60 & 0.86 & $0.66-1.13$ \\
\hline \multicolumn{7}{|c|}{ Chlamydia pneumonia } \\
\hline $\operatorname{Ig} A$ & 73 & 9.07 & 53 & 11.37 & 1.28 & $0.86-1.90$ \\
\hline IgG & 235 & 29.19 & 145 & 31.12 & 1.08 & $0.83-1.40$ \\
\hline \multicolumn{7}{|c|}{ Helicobacter pylori } \\
\hline $\operatorname{IgG}$ & 243 & 30.19 & 182 & 39.06 & 1.39 & $1.08-1.78$ \\
\hline \multicolumn{7}{|c|}{ Cumulative IgA positivity } \\
\hline 0 & 264 & 32.88 & 142 & 30.80 & 1.00 & \\
\hline 1 & 364 & 45.33 & 189 & 41.00 & 1.00 & $0.75-1.33$ \\
\hline 2 & 142 & 17.68 & 112 & 24.30 & 1.47 & $1.05-2.08$ \\
\hline $3-4$ & 33 & 4.11 & 18 & 3.90 & 1.25 & $0.66-2.36$ \\
\hline \multicolumn{7}{|c|}{ Cumulative IgG positivity } \\
\hline $0-1$ & 220 & 27.40 & 125 & 27.11 & 1.00 & \\
\hline 2 & 271 & 33.75 & 160 & 34.71 & 1.09 & $0.80-1.48$ \\
\hline 3 & 227 & 28.27 & 109 & 23.64 & 0.86 & $0.62-1.20$ \\
\hline $4-5$ & 85 & 10.59 & 67 & 14.53 & 1.29 & $0.85-1.95$ \\
\hline \multicolumn{7}{|c|}{ Cumulative overall positivity } \\
\hline $0-1$ & 184 & 22.91 & 103 & 22.34 & 1.00 & \\
\hline 2 & 264 & 32.88 & 148 & 32.10 & 1.03 & $0.74-1.43$ \\
\hline 3 & 235 & 29.27 & 120 & 26.03 & 0.94 & $0.67-1.33$ \\
\hline $4-5$ & 120 & 14.94 & 90 & 19.52 & 1.29 & $0.88-1.90$ \\
\hline
\end{tabular}

Few data missing: in controls: $\mathrm{Mp}, \mathrm{Pg}, \mathrm{Aa}, \mathrm{Cp}, \mathrm{Hp}: \mathrm{n}=4$; in cases: $\mathrm{Mp}, \mathrm{Cp}: 4 ; \mathrm{Pg}$, Aa: $n=9$.

a Conditioned on age (2-year-groups) and sex.
$1.28-3.56$ ) and 3-4 microbial agents (OR 2.50; 95\% CI 1.11-5.65), and IgG-seropositivity for $\mathrm{Hp}$ (OR 1.63; 95\% CI 1.14-2.34) were associated with stroke in age- and sex-adjusted analyses (Fig. 1B; Table 3). Adjustment for SES and further adjustment for cardiovascular risk factors only marginally changed the effect estimates for most seroepidemiological analyses including IgA against $A a$. The association for $C p \operatorname{IgA}$ and $H p$ IgG was weakened and rendered nonsignificant as was the cumulative IgA-seropositivity (two agents: OR 1.88, 95\% CI 0.96-3.69; 3-4 agents: OR $1.5895 \% \mathrm{CI}$ $0.51-4.89)$. $C p$-IgG-seropositivity was associated with higher risk of stroke due to small vessel disease SVD (OR 1.53; 95\% CI 1.04-2.25), an effect that was not found after further adjustment (OR 1.12; 95\% CI 0.67-1.86).

In unadjusted analyses, poor SES in childhood was associated with higher numbers of seropositivities to infectious agents in controls ( $p=0.02$; Jonckhere-test), but particularly in cases $(p=0.0001)$ (Fig. 2). This association was detected for SES in adolescence for cases as well $(p=0.03)$, but not for controls $(p=0.2)$ and did not exist in either group regarding adulthood (cases: $p=0.1$; controls: $p=0.6$ ).

Adjusting for infectious burden had no notable influence on the association between of the risk of stroke and SES in childhood, adolescence, or adulthood (Table 2, model 2). The link between SES in adulthood and stroke risk was reduced and rendered nonsignificant when dental status and frequency of dental health care were additionally adjusted for (Table 2, model 3).

\section{Discussion}

Using a large age- and sex-matched case-control study including detailed data on SES, we found that $A a$-IgA antibody levels were positively, and $M p$-IgG antibody levels were negatively associated with FEIS independent of conventional risk factors and SES. The increased risk found for Aa was mainly due to an association with stroke due to LVD, whose main contributor is atherosclerosis, whereas the inverse association found for $M p$ was not significantly linked to any stroke subtype. The other three microbial agents studied ( $C p, H p$ and $P g$ ) were not independently associated with the overall stroke risk. Cumulative IgA seropositivity was associated with higher risk of FEIS in univariable analysis and after adjustment for SES, but not in the fully adjusted model.

Several serological studies have suggested single chronic infections, as well as the burden composed of several chronic infections as risk factors for stroke [24,25]. The research has mainly focused on $H$. pylori, Cytomegalovirus, Herpes simplex virus, periodontal pathogens, or their combinations [9,10,12,14,26-29]. We investigated the five selected microbes for the following reasons: there is ample evidence that periodontitis increases the risk of stroke and $\mathrm{Aa}$ and $\mathrm{Pg}$ are among the most relevant periodontal agents [30]. We had previously shown that in our region CagA positive $H p$ strains are linked to stroke risk albeit results e.g. from Asia are at variance [11,31]. In addition, we selected $C p$ and $M p$ among respiratory agents as particularly $C p$ previously was in the focus of research.

$P g$ and $A a$ have different characteristics and virulence profiles, but they are both considered to be etiologically linked to periodontitis, which is the sixth most common medical condition according to the Global Burden of Disease Study [32]. Pg is very frequent in chronic adult periodontitis, whereas $A a$ is recurrently encountered in a more aggressive form of the disease, often already starting in adolescence. Both pathogens, however, are very common in adults: according to a Finnish population-based study $\mathrm{Pg}$ and $A a$ are found in $35.5 \%$ and $20.0 \%$ of saliva samples, respectively [33]. In the present study, only $A a$ was associated with increased stroke risk, which is in agreement with an earlier prospective study 

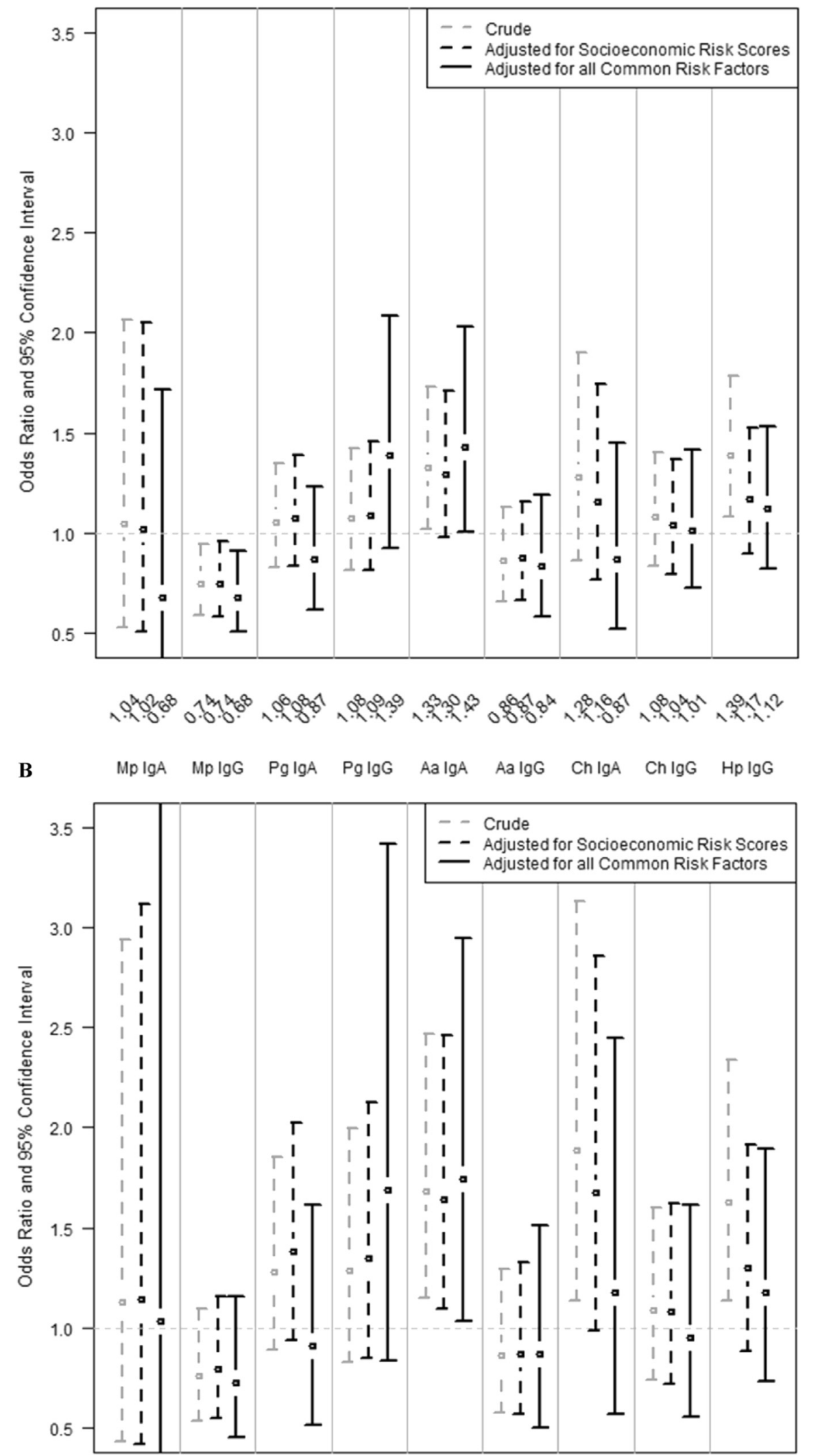

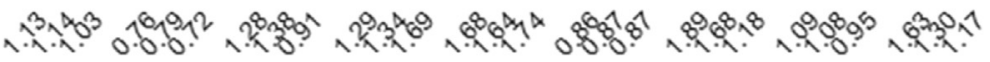

Fig. 1. Odds ratios and 95\%-confidence intervals of ischemic stroke by serology of chronic infections in three different models: (1) conditioned on age and sex, (2) additionally adjusted for socioeconomic risk scores in childhood, adolescence and adulthood, and (3) additionally for all common known risk factors (hypertension, diabetes mellitus, hypercholesterolemia, atrial fibrillation, smoking, (high) alcohol consumption, physical activity, fruit or vegetable consumption, meat consumption, number of teeth, number of dentist visit). (A) Ischemic stroke overall, (B) ischemic stroke due to large vessel disease. 
Table 2

Odds ratios and 95\%-confidence intervals in three multivariable models, for all ischemic stroke cases.

\begin{tabular}{|c|c|c|c|c|c|c|}
\hline & \multicolumn{2}{|c|}{ Model 1} & \multicolumn{2}{|c|}{ Model 2} & \multicolumn{2}{|c|}{ Model 3} \\
\hline & OR & $95 \% \mathrm{CI}$ & OR & $95 \% \mathrm{CI}$ & OR & $95 \% \mathrm{CI}$ \\
\hline \multicolumn{7}{|c|}{ Risk score childhood } \\
\hline Low & 1 & & 1 & & 1 & \\
\hline Middle & 1.47 & $1.04-2.09$ & 1.42 & $1.0-2.02$ & 1.46 & $1.02-2.09$ \\
\hline High & 1.77 & $1.20-2.60$ & 1.72 & $1.16-2.55$ & 1.68 & $1.11-2.52$ \\
\hline \multicolumn{7}{|c|}{ Risk score adolescence } \\
\hline Low & 1 & & 1 & & 1 & \\
\hline Middle & 1.28 & $0.90-1.82$ & 1.26 & $0.89-1.80$ & 1.31 & $0.91-1.88$ \\
\hline High & 1.64 & $0.97-2.78$ & 1.64 & $0.96-2.79$ & 1.62 & $0.93-2.82$ \\
\hline $\begin{array}{l}\text { Risk score adulth } \\
\text { Low }\end{array}$ & \multicolumn{5}{|c|}{ Risk score adulthood } & \\
\hline Middle & 0.89 & $0.62-1.29$ & 0.90 & $0.62-1.31$ & 0.82 & $0.56-1.20$ \\
\hline High & 1.74 & $1.16-2.60$ & 1.73 & $1.15-2.59$ & 1.47 & $0.97-2.23$ \\
\hline \multicolumn{7}{|c|}{ Cumulative IgA positivity } \\
\hline 0 & & & 1 & & 1 & \\
\hline 1 & & & 1.00 & $0.72-1.40$ & 1.03 & $0.73-1.45$ \\
\hline 2 & & & 1.30 & $0.86-1.96$ & 1.32 & $0.86-2.02$ \\
\hline $3-4$ & & & 0.89 & $0.41-1.94$ & 0.77 & $0.33-1.78$ \\
\hline \multicolumn{7}{|c|}{ Cumulative IgG positivity } \\
\hline $0-1$ & & & 1 & & 1 & \\
\hline 2 & & & 1.02 & $0.71-1.47$ & 1.09 & $0.75-1.59$ \\
\hline 3 & & & 0.87 & $0.59-1.30$ & 0.92 & $0.61-1.38$ \\
\hline $4-5$ & & & 0.93 & $0.56-1.54$ & 1.02 & $0.61-1.70$ \\
\hline \multicolumn{7}{|l|}{ No. of teeth } \\
\hline $22-28$ & & & & & 1 & \\
\hline $15-21$ & & & & & 0.87 & $0.55-1.38$ \\
\hline $8-14$ & & & & & 2.33 & $1.41-3.85$ \\
\hline $0-7$ & & & & & 1.07 & $0.75-1.53$ \\
\hline \multicolumn{7}{|l|}{ Dentist visits } \\
\hline$>1 \times$ per year & & & & & 1 & \\
\hline $1 \times$ per year & & & & & 0.94 & $0.69-1.29$ \\
\hline$<1 \times$ per year & & & & & 1.32 & $0.79-2.19$ \\
\hline Never & & & & & 4.47 & $2.41-8.28$ \\
\hline
\end{tabular}

In addition to the depicted parameters all models include age (2-year-groups) sex, hypertension, diabetes mellitus, hypercholesterolemia, atrial fibrillation, coronary heart disease, chronic heart failure, peripheral arterial disease, current smoking, high alcohol consumption, physical activity, fruit/vegetable consumption, meat consumption as published in Ref. [16]. on stroke incidence [9]. In another study, Aa leukotoxin neutralizing antibodies have been associated with decreased risk of stroke further supporting a relevant role of this microbe and its virulence factors in cerebrovascular diseases [34]. Seropositivity to $A a$ does not necessarily denote current periodontitis, but it reflects presence of the pathogen in oral biofilm and the antibody levels are strongly correlated with oral bacterial load [35]. In periodontitis, serum IgA-class antibodies to the pathogen are considered to reflect repeated exposure, which occurs during daily dental routines through bleeding and infected gums. Recurrent bacteraemia, endotoxemia, and spread of antigens lead to low-grade systemic inflammation regardless of the bacterial source [36].

$M p$-seropositivity was found to be associated with the risk of stroke and coronary events in several studies, whereas other studies' results were negative [37,38]. Our study is the first one showing a negative association, which however might be a spurious result.

Our study did not confirm the hypothesis of significant associations between infectious burden measured with cumulative IgAserology, IgG-serology, or aggregated IgG/IgA-positivity to five chronic pathogens, and the overall risk of IS. Heterogeneity of IS etiology might partly explain the study results. Atherosclerosis is thought to play a key role in mechanisms linking chronic infections and IS $[14,39]$. In stroke etiology, atherosclerosis is the main contributor to stroke of LVD origin. Our study showed an association between cumulative IgA-seropositivity and IS in the LVD subgroup, however, this association was weakened after adjustment for all covariates.

We found that disadvantageous childhood socioeconomic conditions reflect increased risk of stroke independent of SES in later life stages and independent of conventional risk factors, including smoking, alcohol consumption, diet and physical activity [16]. The etiology of this association is hardly understood. We had hypothesized that high infectious burden may partly explain this correlation as low SES in childhood increases the risk of infectious diseases including chronic persistent infection with microbial

Table 3

Distribution, odds ratios and 95\%-confidence intervals of serological testing and aggregate number of seropositivities to infectious agents, by TOAST-subgroups.

\begin{tabular}{|c|c|c|c|c|c|c|c|c|c|c|c|c|}
\hline & LVD & $\%$ & Crude $\mathrm{OR}^{\mathrm{a}}$ & $95 \% \mathrm{CI}$ & $\mathrm{CE}$ & $\%$ & Crude $\mathrm{OR}^{\mathrm{a}}$ & $95 \% \mathrm{CI}$ & SVD & $\%$ & Crude $\mathrm{OR}^{\mathrm{a}}$ & $95 \% \mathrm{CI}$ \\
\hline \multicolumn{13}{|c|}{ Mycoplasma pneumonia } \\
\hline IgA & 6 & 3.75 & 1.13 & $0.43-2.94$ & 6 & 5.61 & 2.11 & $0.80-5.58$ & 3 & 2.11 & 0.73 & $0.21-2.55$ \\
\hline IgG & 83 & 51.88 & 0.76 & $0.53-1.09$ & 48 & 44.86 & 0.73 & $0.48-1.11$ & 70 & 49.30 & 0.75 & $0.52-1.08$ \\
\hline \multicolumn{13}{|c|}{ Porphyromonas gingivalis } \\
\hline $\operatorname{Ig} A$ & 97 & 60.62 & 1.28 & $0.89-1.85$ & 59 & 55.14 & 1 & $0.65-1.54$ & 69 & 48.59 & 0.87 & $0.60-1.27$ \\
\hline IgG & 126 & 78.75 & 1.29 & $0.83-2.00$ & 73 & 68.22 & 0.82 & $0.50-1.33$ & 104 & 73.24 & 1.10 & $0.71-1.69$ \\
\hline \multicolumn{13}{|c|}{ Aggregatibacter actinomycem } \\
\hline $\operatorname{Ig} A$ & 59 & 36.88 & 1.68 & $1.15-2.46$ & 34 & 31.78 & 1.29 & $0.81-2.04$ & 38 & 26.76 & 1.05 & $0.68-1.60$ \\
\hline IgG & 41 & 25.62 & 0.86 & $0.58-1.29$ & 30 & 28.04 & 1.04 & $0.65-1.66$ & 32 & 22.54 & 0.72 & $0.46-1.11$ \\
\hline \multicolumn{13}{|c|}{ Chlamydia pneumonia } \\
\hline $\operatorname{Ig} A$ & 26 & 16.25 & 1.89 & $1.14-3.13$ & 8 & 7.48 & 0.82 & $0.38-1.80$ & 13 & 9.15 & 0.99 & $0.52-1.89$ \\
\hline $\operatorname{IgG}$ & 52 & 32.5 & 1.09 & $0.74-1.60$ & 26 & 24.3 & 0.85 & $0.52-1.38$ & 54 & 38.03 & 1.53 & $1.04-2.25$ \\
\hline \multicolumn{13}{|c|}{ Helicobacter pylorii } \\
\hline $\operatorname{IgG}$ & 71 & 44.38 & 1.63 & $1.14-2.34$ & 41 & 38.32 & 1.30 & $0.84-2.01$ & 49 & 34.51 & 1.18 & $0.79-1.74$ \\
\hline \multicolumn{13}{|c|}{ Cumulative IgA positivity } \\
\hline 0 & 38 & 23.75 & 1 & & 29 & 27.10 & 1 & & 54 & 38.03 & 1 & \\
\hline 1 & 64 & 40 & 1.31 & $0.83-2.07$ & 43 & 40.19 & 0.94 & $0.56-1.59$ & 54 & 38.03 & 0.75 & $0.49-1.15$ \\
\hline 2 & 47 & 29.38 & 2.14 & $1.28-3.56$ & 29 & 27.10 & 1.58 & $0.87-2.86$ & 27 & 19.01 & 0.93 & $0.54-1.58$ \\
\hline $3-4$ & 10 & 6.25 & 2.5 & $1.11-5.65$ & 2 & 1.87 & 0.56 & $0.12-2.55$ & 5 & 3.52 & 0.95 & $0.34-2.66$ \\
\hline \multicolumn{13}{|c|}{ Cumulative IgG positivity } \\
\hline $0-1$ & 35 & 21.88 & 1 & & 33 & 30.84 & 1 & & 38 & 26.76 & 1 & \\
\hline 2 & 57 & 35.62 & 1.23 & $0.77-1.98$ & 33 & 30.84 & 0.86 & $0.50-1.46$ & 47 & 33.10 & 1.09 & $0.67-1.75$ \\
\hline 3 & 39 & 24.38 & 0.98 & $0.59-1.64$ & 22 & 20.56 & 0.66 & $0.36-1.19$ & 39 & 27.46 & 1.07 & $0.65-1.76$ \\
\hline $4-5$ & 28 & 17.5 & 1.66 & $0.91-3.01$ & 15 & 14.02 & 1.22 & $0.60-2.46$ & 16 & 11.27 & 1.04 & $0.53-2.04$ \\
\hline \multicolumn{13}{|c|}{ Cumulative overall positivity } \\
\hline $0-1$ & 28 & 17.5 & 1 & & 26 & 24.3 & 1 & & 34 & 23.94 & 1 & \\
\hline 2 & 48 & 30 & 1.08 & $0.64-1.81$ & 36 & 33.64 & 0.97 & $0.55-1.70$ & 43 & 30.28 & 0.96 & $0.58-1.59$ \\
\hline 3 & 42 & 26.25 & 1.08 & $0.63-1.85$ & 24 & 22.43 & 0.73 & $0.40-1.35$ & 41 & 28.87 & 1.01 & $0.61-1.69$ \\
\hline $4-5$ & 41 & 25.62 & 1.83 & $1.04-3.22$ & 17 & 15.89 & 0.95 & $0.47-1.90$ & 22 & 15.49 & 1 & $0.54-1.86$ \\
\hline
\end{tabular}

${ }^{\text {a }}$ Conditioned on age (2-year-groups) and sex. 


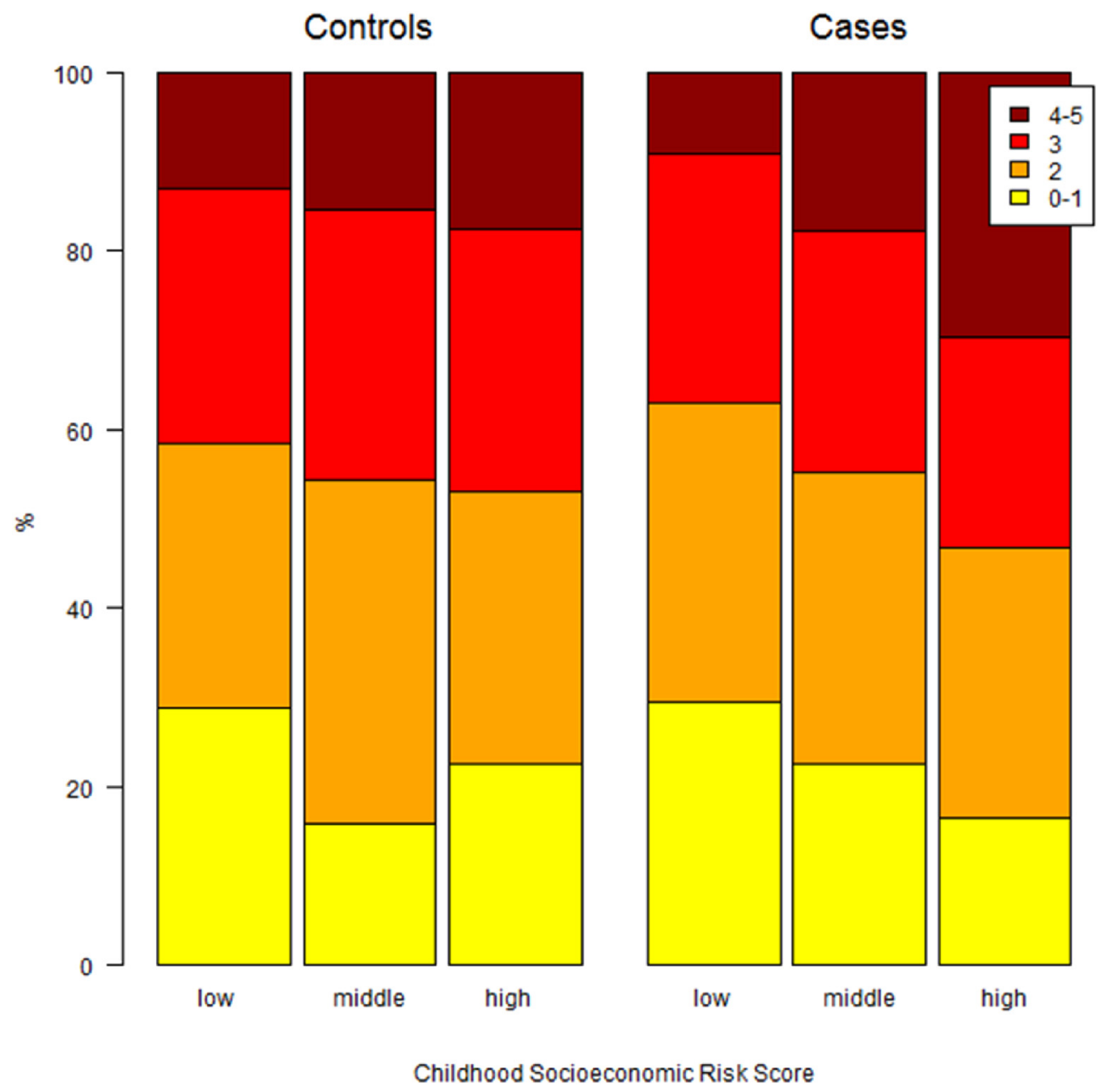

Fig. 2. Socioeconomic risk score in childhood by combined IgA/IgG seropositivity.

agents such as $H p$ [17]. In fact we found a strong correlation between aggregate burden of infections and SES in childhood in cases, and to a weaker degree in adolescence. In controls, such association was less pronounced but significant in childhood and not existent in adolescence. These findings are in line with previous results. However, addition of serological data only slightly attenuated the effect of childhood socioeconomic status on stroke. Thus, the effect of presently examined infections on the association between childhood SES and IS is low.

Interestingly, advanced tooth loss (8-14 remaining teeth) and avoidance of dental health care service were strongly and independently associated with FEIS. Inclusion of the number of teeth and the frequency of dentist visits reduced the strength of the association between low SES in adulthood and stroke, and rendered it nonsignificant. This indicates that behavioral and other factors that are reflected by dental care or the sequelae of dental negligence contribute to the link between SES and stroke, a finding that has not been reported so far to the best of our knowledge. Of note, periodontitis, a chronic infectious disease that is influenced by several behavioral (e.g. smoking) and socioeconomic factors, is among the most common contributors to the loss of teeth.

Our study has several limitations including the low number of microbial agents assessed, the low number of subjects in etiologic stroke subgroups a low response rate in controls and the fact that diagnosis of prior MI (>90 days) was not analyzed separately. In addition, the individual reason for missing teeth was unknown.
Strengths of our study include the ample information on SES in different life periods and on other stroke risk factors.

In conclusion, we could not find that infectious burden as assessed with five microbial agents independently contributes to stroke risk in a consistent way. Low SES in childhood is associated with stroke risk and with a higher number of antimicrobial seropositivities. However, this elevated infectious burden does not contribute to our understanding of the link between childhood conditions and stroke. Advanced tooth loss due to dental negligence appears to be part of a lifestyle that contributes to the association between disadvantageous adulthood SES and FEIS.

\section{Conflict of interest}

The authors declared they do not have anything to disclose regarding conflict of interest with respect to this manuscript.

\section{Financial support}

This work was supported by the German Research Foundation (DFG), [BE 2056/5-1, GR 1102/5-1, GR 1687/2-1], the Academy of Finland (grant number 1266053), the Finnish Dental Association Apollonia and the Yrjö Jahnsson foundation. 


\section{Appendix A. Supplementary data}

Supplementary data related to this article can be found at http:// dx.doi.org/10.1016/j.atherosclerosis.2016.10.008.

\section{References}

[1] V.L. Feigin, G.A. Roth, M. Naghavi, P. Parmar, R. Krishnamurthi, S. Chugh, et al, Global burden of stroke and risk factors in 188 countries, during 1990-2013: a systematic analysis for the Global Burden of Disease Study 2013, Lancet Neurol. 15 (2016) 913-924.

[2] F. Palm, M. Dos Santos, C. Urbanek, M. Greulich, K. Zimmer, A. Safer, et al. Stroke seasonality associations with subtype, etiology and laboratory results in the Ludwigshafen Stroke Study (LuSSt), Eur. J. Epidemiol. 28 (2013) $373-381$.

[3] Y. Liao, K.J. Greenlund, J.B. Croft, N.L. Keenan, W.H. Giles, Factors explaining excess stroke prevalence in the US Stroke Belt, Stroke 40 (2009) 3336-3341.

[4] A.J. Grau, L. Marquardt, C. Lichy, The effect of infections and vaccinations on stroke risk, Expert Rev. Neurother. 6 (2006) 175-183.

[5] A.J. Grau, C. Urbanek, F. Palm, Common infections and the risk of stroke, Nat. Rev. Neurol. 6 (2010) 681-694.

[6] B. Galobardes, G.D. Smith, J.W. Lynch, Systematic review of the influence of childhood socioeconomic circumstances on risk for cardiovascular disease in adulthood, Ann. Epidemiol. 16 (2006) 91-104.

[7] A. Flex, E. Gaetani, P. Papaleo, G. Straface, A.S. Proia, G. Pecorini, et al, Proinflammatory genetic profiles in subjects with history of ischemic stroke, Stroke 35 (2004) 2270-2275.

[8] A.J. Grau, H. Becher, C.M. Ziegler, C. Lichy, F. Buggle, C. Kaiser, et al., Periodontal disease as a risk factor for ischemic stroke, Stroke 35 (2014) 496-501.

[9] P.J. Pussinen, G. Alfthan, H. Rissanen, A. Reunanen, S. Asikainen, P. Knekt, Antibodies to periodontal pathogens and stroke risk, Stroke 35 (2004) 2020-2023.

[10] P.J. Pussinen, G. Alfthan, P. Jousilahti, S. Paju, J. Tuomilehto, Systemic exposure to Porphyromonas gingivalis predicts incident stroke, Atherosclerosis 193 (2007) 222-228.

[11] M.R. Preusch, A.J. Grau, F. Buggle, C. Lichy, J. Bartel, C. Black, et al., Association between cerebral ischemia and cytotoxin-associated gene-A-bearing strains of Helicobacter pylori, Stroke 35 (2004) 1800-1804.

[12] J. Ngeh, C. Goodbourn, Chlamydia pneumoniae, Mycoplasma pneumoniae, and Legionella pneumophila in elderly patients with stroke (C-PEPS, M-PEPS, L-PEPS): a case-control study on the infectious burden of atypical respiratory pathogens in elderly patients with acute cerebrovascular disease, Stroke 36 (2005) 259-265.

[13] P. Apfalter, Chlamydia pneumoniae, stroke, and serological associations: anything learned from the atherosclerosis-cardiovascular literature or do we have to start over again? Stroke 37 (2006) 756-758.

[14] M.S. Elkind, P. Ramakrishnan, Y.P. Moon, B. Boden-Albala, K.M. Liu S.L. Spitalnik, et al., Infectious burden and risk of stroke: the northern Manhattan study, Arch. Neurol. 67 (2010) 33-38.

[15] J. Addo, L. Ayerbe, K.M. Mohan, S. Crichton, A. Sheldenkar, R. Chen, et al, Socioeconomic status and stroke: an updated review, Stroke 43 (2012) 1186-1191.

[16] H. Becher, F. Palm, A. Aigner, A. Safer, C. Urbanek, F. Buggle, et al., Socioeconomic conditions in childhood, adolescence, and adulthood and the risk of ischemic stroke, Stroke 47 (2016) 173-179.

[17] L.J. Murray, E.E. McCrum, A.E. Evans, K.B. Bamford, Epidemiology of Helicobacter pylori infection among 4742 randomly selected subjects from Northern Ireland, Int. J. Epidemiol. 26 (1997) 880-887.

[18] F. Palm, C. Urbanek, S. Rose, F. Buggle, B. Bode, M.G. Hennerici, et al., Stroke incidence and survival in Ludwigshafen am Rhein, Germany: the Ludwigshafen stroke study (LuSSt), Stroke 41 (2010) 1865-1870.

[19] P.J. Pussinen, T. Vilkuna-Rautiainen, G. Alfthan, K. Mattila, S. Asikainen, Multiserotype enzyme-linked immunosorbent assay as a diagnostic aid for periodontitis in large-scale studies, J. Clin. Microbiol. 40 (2002) 512-518.

[20] F. Palm, C. Urbanek, J. Wolf, F. Buggle, T. Kleemann, M.G. Hennerici, et al., Etiology, risk factors and sex differences in ischemic stroke in the Ludwigshafen Stroke Study, a population-based stroke registry, Cerebrovasc. Dis. 33 (2012) 69-75.

[21] R RCT, A Language and Environment for Statistical Computing, R Foundation for Statistical Computing, Vienna, Austria, 2013.

[22] T T. A Package for Survival Analysis in S. R Package Version 2.37-7, 2014. http://CRANR-projectorg/package=survival.

[23] E V Clinfun: Clinical Trial Design and Data Analysis Functions. R Package Version 1.0.11. http://CRANR-projectorg/package=clinfun.

[24] A. Paganini-Hill, E. Lozano, G. Fischberg, M. Perez Barreto, K. Rajamani, S.F. Ameriso, et al., Infection and risk of ischemic stroke: differences among stroke subtypes, Stroke 34 (2003) 452-457.

[25] P.J. Lindsberg, A.J. Grau, Inflammation and infections as risk factors for ischemic stroke, Stroke 34 (2003) 2518-2532.

[26] B. Schottker, M.A. Adamu, M.N. Weck, H. Muller, H. Brenner, Helicobacter pylori infection, chronic atrophic gastritis and major cardiovascular events: a population-based cohort study, Atherosclerosis 220 (2012) 569-574.

[27] N. Hosomi, S. Aoki, K. Matsuo, K. Deguchi, H. Masugata, K. Murao, et al., Association of serum anti-periodontal pathogen antibody with ischemic stroke, Cerebrovasc. Dis. 34 (2012) 385-392.

[28] A.W. Haider, P.W. Wilson, M.G. Larson, J.C. Evans, E.L. Michelson, P.A. Wolf, et al., The association of seropositivity to Helicobacter pylori, Chlamydia pneumoniae, and cytomegalovirus with risk of cardiovascular disease: a prospective study, J. Am. Coll. Cardiol. 40 (2002) 1408-1413.

[29] S. Sealy-Jefferson, B.W. Gillespie, A.E. Aiello, M.N. Haan, L.B. Morgenstern, L.D. Lisabeth, Antibody levels to persistent pathogens and incident stroke in Mexican Americans, PLoS One 8 (2013) e65959.

[30] A.D. Haffajee, M.A. Cugini, A. Tanner, R.P. Pollack, C. Smith, R.L. Kent, et al., Subgingival microbiota in healthy, well-maintained elder and periodontitis subjects, J. Clin. Periodontol. 25 (1998) 346-353.

[31] A. Ikeda, H. Iso, S. Sasazuki, M. Inoue, S. Tsugane, The combination of Helicobacter pylori- and cytotoxin-associated gene-A seropositivity in relation to the risk of myocardial infarction in middle-aged Japanese: the Japan Public Health Center-based study, Atherosclerosis 230 (2013) 67-72.

[32] M. Desvarieux, R.T. Demmer, D.R. Jacobs, P.N. Papapanou, R.L. Sacco, T. Rundek, Changes in clinical and microbiological periodontal profiles relate to progression of carotid intima-media thickness: the Oral Infections and Vascular Disease Epidemiology study, J. Am. Heart Assoc. 2 (2013) e000254.

[33] F. Kononen, S. Paju, PJ. Pussinen, M. Hyvonen, P. Di Tella, L. Suominen-Taipale, et al., Population-based study of salivary carriage of periodontal pathogens in adults, J. Clin. Microbiol. 45 (2007) 2446-2451.

[34] A. Johansson, I. Johansson, M. Eriksson, A.M. Ahren, G. Hallmans, B. Stegmayr, Systemic antibodies to the leukotoxin of the oral pathogen Actinobacillus actinomycetemcomitans correlate negatively with stroke in women, Cerebrovasc. Dis. 20 (2005) 226-232.

[35] P.J. Pussinen, E. Kononen, S. Paju, K. Hyvarinen, U.K. Gursoy, S. Huumonen, et al., Periodontal pathogen carriage, rather than periodontitis, determines the serum antibody levels, J. Clin. Periodontol. 38 (2011) 405-411.

[36] T.G. Glaros, S. Chang, E.A. Gilliam, U. Maitra, H. Deng, L. Li, Causes and consequences of low grade endotoxemia and inflammatory diseases, Front. Biosci. Sch. Ed. 5 (2013) 754-765.

[37] C.H. Chiang, C.C. Huang, W.L. Chan, Y.C. Chen, T.J. Chen, S.J. Lin, et al., Association between Mycoplasma pneumonia and increased risk of ischemic stroke: a nationwide study, Stroke 42 (2011) 2940-2943.

[38] J. Ngeh, S. Gupta, C. Goodbourn, G. McElligott, Mycoplasma pneumoniae in elderly patients with stroke. a case-control study on the seroprevalence of $\mathrm{M}$. pneumoniae in elderly patients with acute cerebrovascular disease - the MPEPS Study, Cerebrovasc. Dis. 17 (2004) 314-319.

[39] S. Kiechl, G. Egger, M. Mayr, C.J. Wiedermann, E. Bonora, F. Oberhollenzer, et al., Chronic infections and the risk of carotid atherosclerosis: prospective results from a large population study, Circulation 103 (2001) 1064-1070. 